\title{
Size-assortative mating in simultaneous hermaphrodites: an experimental test and a meta-analysis
}

\author{
Stuart Graham $^{1} \cdot$ Elodie Chapuis $^{1,2} \cdot$ Stefania Meconcelli ${ }^{1,3} \cdot$ Nicolas Bonel $^{1,4} \cdot$ \\ Kevin Sartori $^{1} \cdot$ Ananda Christophe $^{1} \cdot$ Pilar Alda $^{5} \cdot$ Patrice David $^{1} \cdot$ Tim Janicke $^{1}(\mathbb{D})$
}

Received: 1 June 2015 /Revised: 18 August 2015 / Accepted: 24 August 2015 / Published online: 5 September 2015

(C) Springer-Verlag Berlin Heidelberg 2015

\begin{abstract}
Assortative mating by size has been argued to be widespread in the animal kingdom. However, the strength of size-assortative mating is known to vary considerably between species and the underlying mechanisms promoting this inter-specific variation remain largely unexplored. Sizeassortative mating has been proposed to be particularly strong in simultaneous hermaphrodites, i.e. organisms that produce male and female gametes at the same time. Here, we build on this hypothesis by arguing that size-assortative mating mediated by sexual selection is generally stronger in reciprocally mating hermaphrodites compared with unilaterally mating species and separate-sexed organisms. We report a series of
\end{abstract}

Communicated by J. C. Choe

Electronic supplementary material The online version of this article (doi:10.1007/s00265-015-1999-5) contains supplementary material, which is available to authorized users.

Tim Janicke

janicke.tim@gmail.com

1 Centre d'Ecologie Fonctionnelle et Evolutive, UMR 5175, CNRS, Université de Montpellier, Université Paul-Valéry Montpellier, Ecole Pratique des Hautes Etudes, 1919 Route de Mende, 34293 Montpellier Cedex 05, France

2 IRD, UMR186 "Intéractions Plantes-MicrorganismesEnvironement”, 911, Avenue Agropolis, BP 64501, 34394 Montpellier Cedex 05, France

3 Department of Life Sciences and Systems Biology, Università di Torino, Turin, Italy

4 Laboratorio de Zoología de Invertebrados I, Departamento de Biología, Bioquímica y Farmacia, Universidad Nacional del Sur, Bahía Blanca, Argentina

5 Centro de Estudios Parasitológicos y de Vectores (CEPAVE, CCT-La Plata-CONICET-UNLP), Avenida 120 s/n e/61 y 62, 1900 La Plata, Buenos Aires, Argentina empirical tests suggesting that size-assortative mating in the unilaterally copulating freshwater snail Physa acuta is caused by spatial clustering of similar-sized individuals and not by mate choice. In addition, we present a meta-analysis testing, for the first time, the hypothesis that sexual selection-mediated size-assortative mating is stronger in reciprocally copulating simultaneous hermaphrodites. Overall, we found significant size-assortative mating across 18 tested species and substantial inter-specific variation. Importantly, part of this variation can be explained by mating type, providing support for the hypothesis that size-assortative mating is stronger in reciprocally mating hermaphrodites compared with unilaterally mating species. We highlight potential pitfalls when testing for sexual selection-mediated size-assortative mating and discuss the need for more experimental and comparative approaches in order to resolve the observed variation in the strength of size-assortative mating among species.

Keywords Assortative mating $\cdot$ Body size $\cdot$ Density $\cdot$ Female fecundity $\cdot$ Mate choice $\cdot$ Physa acuta $\cdot$ Sexual selection

\section{Introduction}

Assortative mating, the non-random formation of mating pairs based on similarity, has been argued to have important evolutionary implications because of its role in promoting speciation and maintaining standing genetic variation within populations (e.g. Seehausen et al. 1997; Kondrashov and Shpak 1998; Lynch and Walsh 1998; Kirkpatrick 2000). Many alternative hypotheses have been proposed to explain assortative mating. In a review of the most prominent of these, Crespi (1989) distinguished three classes of hypotheses on the basis of the mechanisms which they rely on: (i) sexual selection, (ii) mate availability and (iii) constraints. First, sexual selection is 
expected to generate assortative mating if both sexes have a mutual mating preference for a given phenotype or if the mating preference for a given phenotype is expressed by only one sex and the preferred phenotype also confers a mating advantage for the choosy sex (e.g. Harari et al. 1999). Here, a mating advantage represents an improved ability to outcompete rivals and/or to overcome resistance to mating exerted by a potential partner. Importantly, sexual selection can only promote sizeassortative mating if the individuals with less desirable/ competitive phenotypes accept less desirable mating partners irrespective of their preference. Second, heterogeneity in mate availability, such that individuals expressing similar phenotypes are either spatially or temporally clustered, may lead to assortative mating in the absence of mate choice (e.g. Ferrer and Penteriani 2003). And third, physical constraints that prevent successful copulation between dissimilar individuals could also lead to assortative mating (e.g. Han et al. 2010).

Assortative mating has been found to involve many different traits, including visual signals, structural characters, age and various predictors of condition (Jiang et al. 2013). However, by far the best studied trait associated with assortative mating is body size (e.g. Arnqvist et al. 1996; Hoefler 2007; Baldauf et al. 2009; Mobley et al. 2014). Given the classification of Crespi (1989), in the absence of any size-based heterogeneity in mate availability or physical constraints, sizeassortative mating is considered to be the outcome of sexual selection. In the majority of cases, size-based mating preferences manifest as a preference for larger mates. Thus, sizeassortative mating is expected to result from either: (i) a mutual mating preference by both sexes for larger mates or (ii) a preference for larger mates in only one sex combined with a mating advantage of larger individuals in the choosy sex.

Size-assortative mating has been documented for a broad range of animal taxa including insects, birds, reptiles and mammals, and a recent meta-analysis revealed substantial variation in the strength of size-assortative mating between species (Jiang et al. 2013). The authors speculated that this variation could reflect differences in mating systems or other lifehistory traits among species and highlighted the need for comparative approaches to uncover the evolutionary forces promoting size-assortative mating. One potential source of the observed inter-specific variation is the mode of how the sexes are expressed. Specifically, size-assortative mating that is mediated by sexual selection has been proposed to be particularly pronounced in simultaneous hermaphrodites (hereafter hermaphrodites), i.e. organisms that produce male and female gametes at the same time (Michiels 1998). Michiels (1998) argued that a preference for mating with larger partners in only one sex function could lead to size-assortative mating in hermaphrodites even in the absence of a corresponding mating advantage of the choosy sex because all individuals will express the preference. More recently, Anthes (2010) discussed the hypothesis of Michiels (1998) specifically with respect to reciprocally mating hermaphrodites (i.e. those which both donate and receive sperm in each mating encounter) without clarifying whether it also applies to unilaterally mating hermaphrodites (i.e. those where each individual of the mating pair can only donate or receive sperm). Here, we build on the work of Michiels (1998) and Anthes (2010) and present a clear hypothesis of how the strength of size-assortative mating can be expected to differ between unilaterally and reciprocally mating hermaphrodites.

As discussed above, whenever there is a preference for larger partners in both sexes, or a preference for larger partners in one sex coupled with a mating advantage of larger individuals in the choosy sex, we expect size-assortative mating in separate-sexed species and hermaphrodites alike. However, a preference for larger partners may exist only in one sex function and body size may provide no mating advantage. In particular, the male function is often expected to benefit from mating with larger sperm recipients because of the widely documented positive correlation between body size and female fecundity (e.g. Honek 1993; Anthes 2010); whereas, a female preference for large sperm donors is less well documented in hermaphrodite taxa (e.g. Ohbayashi-Hodoki et al. 2004). Therefore, we will now explain our expectations for the strength of size-assortative mating in hermaphrodites and separate-sexed species when the preference for larger partners is only beneficial to the male sex function; although a symmetrical reasoning would apply to cases where only the female function benefits from mating with larger partners.

To begin with, it is important to realise that selection in simultaneous hermaphrodites (i.e. reciprocally and unilaterally mating species) does not act independently on the male and female sex, as is the case for separate-sexed organisms, but instead on the fitness of the whole organism. Under the abovementioned assumptions, for both separate-sexed organisms and unilaterally mating hermaphrodites, mating with bigger individuals will only be beneficial for individuals acting as a male. As a consequence, only half of the copulating individuals will select partners based on their body size, preventing size-assortative mating. In unilaterally mating hermaphrodites, accidental encounters between two similar-sized individuals may even promote mating conflicts (reviewed in Schärer et al. 2014). Specifically, sex-role preferences in unilaterally mating hermaphrodites may be size-dependent meaning that similar-sized individuals insist on adopting the same sex role (e.g. all small individuals have a preference for mating as male; Ohbayashi-Hodoki et al. 2004; Nakadera et al. 2015). Such conflicting sex-role preferences of similar-sized individuals may arise from size-dependent sex allocation where small individuals typically have a more male-biased sex allocation (reviewed in Schärer 2009), which has been argued to translate into a preference for male sex role (Anthes et al. 2006b; Janicke and Schärer 2009). If these conflicts cannot be resolved (e.g. by gamete trading; Fischer 1984), size-assorted 
pairings may be prevented. By contrast, in reciprocally mating hermaphrodites, it is beneficial for all copulating individuals to reject small partners, simply because all individuals donate sperm in every mating event. This should lead to a populationwide preference for larger partners and, as a consequence, to size-assortative mating. Moreover, conflict over the mating roles is resolved in reciprocal mating hermaphrodites as both mates can copulate in their preferred sex role (Schärer et al. 2014).

Overall, we hypothesise that size-assortative mating that is mediated by sexual selection will be stronger in reciprocally mating hermaphrodites than in unilaterally mating hermaphrodites and separate-sexed species. Further, we propose that the strength of size-assortative mating in unilaterally mating hermaphrodites relative to separate-sexed species will depend on the strength of conflict over the sex roles. Size-dependent sex-role preferences will lead to mating conflicts and weaker size-assortative mating in unilaterally mating hermaphrodites compared with separate-sexed species.

Empirical evidence for size-assortative mating in hermaphrodites is equivocal, with some studies demonstrating strong size-assortative mating (e.g. Petersen and Fischer 1996; Vreys and Michiels 1997; Clarke and Fields 2013) and others suggesting pair formation to be random with respect to size (e.g. Michiels et al. 2001; Chaine and Angeloni 2005; Koene et al. 2007). Until now, it remains unclear whether these contrasting findings reflect methodological differences of how sizeassortative mating has been tested or whether the observed inter-specific variation can be explained by the mating types (i.e. reciprocal or unilateral) of the studied species.

This study has two principal aims. The first is to highlight some of the potential methodological pitfalls associated with testing for sexual selection-mediated size-assortative mating, such as the failure to account for spatial and/or temporal segregation of individuals based on body size. To do this, we tested for size-assortative mating in the unilaterally mating hermaphroditic freshwater snail Physa acuta using three different approaches: (i) a fully descriptive field study, (ii) an experimental test on freshly field-caught snails and (iii) an experimental test using size-manipulated snails under laboratory conditions. Experimental tests of size-dependent mating behaviour in $P$. acuta suggest that sperm donors have a preference to inseminate larger partners (Ohbayashi-Hodoki et al. 2004). However, explicit tests for size-assortative mating are currently lacking for this species (even though the data presented in two studies allow it to be tested for post hoc; see meta-analysis). The second aim of this study is to test whether our current knowledge on pair formation in hermaphrodite species supports our hypothesis that size-assortative mating is stronger in reciprocally mating than unilaterally mating species. To achieve this we conducted a meta-analysis of published tests for sizeassortative mating.

\section{Methods}

\section{Size-assortative mating in the hermaphroditic freshwater snail $P$. acuta}

\section{Study organism}

P. acuta is a simultaneously hermaphroditic freshwater snail known to be a preferential outcrosser (e.g. David et al. 2007) with self-fertilisation leading to severe inbreeding depression in both sex functions (Janicke et al. 2013). These snails copulate unilaterally, and copulations are always initiated by the male-acting snail, which crawls onto the shell of the partner until it is mounted in a position that allows insertion of its phallus into the partner's gonopore (Wethington and Dillon 1996). In the laboratory, snails are usually kept at $25{ }^{\circ} \mathrm{C}$ in small plastic boxes $(200 \mathrm{~mL})$ and fed with boiled lettuce. Under these conditions, snails mature within 6-8 weeks and adults lay a gelatinous egg capsule containing several tens of eggs every 1-2 days.

Body size has been shown to be a good predictor of female fecundity in P. acuta (e.g. Pélissié et al. 2012; Janicke et al. 2013). In this study, we used either shell length (aperture to apex; measured to the nearest $0.1 \mathrm{~mm}$ using callipers) or body weight (measured to the nearest $0.1 \mathrm{mg}$ using a Mettler PM100 balance, Mettler-Toledo, Greifensee, Switzerland) as estimates of body size. Preliminary experiments revealed that these two measures are highly correlated (measures taken from 80 lab-reared adult individuals; Pearson correlation: $r=$ $0.929, P<0.001)$ and that both of them can be measured with high repeatability (intra-class correlation coefficients $\left(r_{i}\right.$; Lessells and Boag 1987) for body length and weight based on measurements taken twice from 50 and 30 lab-reared adult individuals, respectively; shell length: $r_{\mathrm{i}}=0.980, F_{49},{ }_{50}=$ 97.654, $P<0.001$; body weight: $r_{\mathrm{i}}=0.986, F_{29}, 30=144.267$, $P<0.001)$.

\section{Size-assortative mating in the field}

We tested for size-assortative mating in the field by sampling copulating pairs along a 200-m-long stretch of the river Lez $\left(50^{\circ} 55,6^{\prime} \mathrm{N}, 11^{\circ} 34,6^{\prime} \mathrm{E}\right)$, located $15 \mathrm{~km}$ north of Montpellier (France) on 29 May 2014. The sampling area was divided into four sectors (each sector approximately $50 \mathrm{~m}$ long), which allowed us to control statistically for spatial variation in body size at this arbitrarily chosen scale. For each individual, we measured shell length and noted the sex role and sector in which it was found mating. It should be noted that in the field, it is often impossible to check whether the male acting snail is actually inseminating its partner. Therefore, we considered each encounter in which the male acting snail was mounting close to the female gonopore of its partner as a copulation. In total, we sampled 68 pairs (sample sizes for sectors A to D: 
$n_{\mathrm{A}}=11, n_{\mathrm{B}}=31, n_{\mathrm{C}}=14, n_{\mathrm{D}}=12$ ) and tested for size-assortative mating by computing $r_{\mathrm{i}}$ using raw estimates and standardised estimates of shell length. Raw estimates were standardised by subtracting from them the mean shell length of all individuals observed copulating in the same sector, and then dividing these resulting values by the standard deviation of shell length of all individuals observed copulating in the sector. The standardised estimates therefore allowed us to control statistically for spatial variation in body size.

\section{Size-assortative mating under standardised conditions}

In order to test for sexual selection-mediated size-assortative mating under conditions that control for spatial variation in body size, we sampled snails in the field, assigned them to groups of ten individuals and observed their mating behaviour in tanks under two different densities, assuring that all individuals had access to partners of all size classes observed in the field. We tested for the effect of density on size-assortative mating because density has been argued to be an important factor influencing the strength and direction of pre-copulatory sexual selection (Kokko and Rankin 2006). More specifically, density may affect size-assortative mating by: (i) facilitating or impeding the monopolisation of bigger mating partners by bigger individuals and/or (ii) altering the net benefits of mate choice as a consequence of its effect on mate searching costs.

In detail, we first collected 120 snails from the same field site, and on the same date, as our test of size-assortative mating in the field (see above). Next, we measured the shell length of all snails and marked them individually using Edding 751 gloss paint marker (Edding AG, Ahrensburg, Germany). Previous studies have indicated that this marking method does not affect mating behaviour of either sex function (Henry and Jarne 2007; Janicke et al. 2014). Then, we assigned each individual to one of 12 groups containing 10 individuals in a way that maximised the variance in body size within groups while keeping the mean and the variance in body size constant among groups. Finally, we performed mating trials that lasted $60 \mathrm{~min}$, noting the identity of both snails involved in each copulation. During the mating trials, each group was kept at one of two different densities by using plastic tanks that differed in volume. Specifically, we filled tanks of two different volumes (i.e. six big and six small tanks) with water taken directly from their natural habitat up to a level of $3 \mathrm{~cm}$. Using these differently sized tanks, we induced a 2.5-fold difference in the available underwater surface area (i.e. underwater surface area of low-density tanks, $640 \mathrm{~cm}^{2}$; underwater surface area of high-density tanks, $260 \mathrm{~cm}^{2}$ ). As the movements of $P$. acuta are restricted to the benthic substrate, we propose that surface area provides a sensible means for the manipulation of density while keeping the number of individuals per tank constant. During processing (period from sampling until the start of the mating trials), all individuals were kept in isolation for
$3 \mathrm{~h}$, which ensured that snails could recover from handling. All observations were carried out by three observers, who each recorded the mating behaviour of four tanks (i.e. two low-density and two high-density) simultaneously.

As intended by our experimental design, the mean and the variance of shell length did not differ between tanks (mean comparison: one-way analysis of variance (ANOVA), $F_{11}$, ${ }_{108}=0.014, P>0.999$; variance comparison: Levene's test, $\left.F_{11,108}=0.119, P>0.999\right)$. In addition, neither the number of encounters nor the number of copulations differed between tanks (one-way ANOVA: all $P>0.5$ ), and therefore, we pooled the data from all 6 tanks of each density treatment for the final analysis. Specifically, we tested for size-assortative mating by computing $r_{\mathrm{i}}$ separately for each density treatment.

\section{Size-assortative mating of experimentally manipulated snails}

In a third empirical test of size-assortative mating in P. acuta, we experimentally manipulated body size and tested whether this affected pair formation under laboratory conditions. This was done in order to narrow down potential factors that are confounded with body size. In particular, age is typically correlated with body size and has been shown to affect mating strategies in simultaneous hermaphrodites (Hermann et al. 2009; Nakadera et al. 2015). We pooled adult snails from an outbred laboratory stock culture $(n=33)$ in a large plastic tank and let them lay eggs for 4 days. At an age of 27 days after hatching, 72 randomly sampled juveniles were isolated and divided equally between two feeding regimes: snails in the first treatment were fed ad libitum throughout growth until the mating trials started (hereafter 'well-fed') and those in the second treatment were exposed to an alternating feeding regime of providing food ad libitum and no food (hereafter 'poorly fed') with the alternations occurring every 2 or 3 days (mean \pm SE, $2.40 \pm 0.16$ days). At an age of 55 days, we paired all individuals with either an equally or differently treated individual (i.e. 18 homogeneous pairs, 18 heterogeneous pairs) for $1 \mathrm{~h}$. This was done to test for body size-dependent copulatory investment, which was the focus of another study that is not reported here. After these mating trials, all individuals were isolated to let them lay eggs for 3 days, which allowed us to test for an effect of the food treatment on female fecundity, estimated in terms of the number of eggs. After egg laying, at an age of 65 days, we assessed body weight of all individuals and performed mating trials to test for sizeassortative mating on the following day. For this, we created groups of four individuals consisting of two randomly chosen well-fed and two poorly fed individuals, which were individually marked using Edding 751 gloss paint marker (Edding AG, Ahrensburg, Germany; see above). Mating trials were carried out in glass beakers $(350 \mathrm{~mL})$ filled with spring water and lasted for 60 min during which all copulations were recorded. In total, we observed 18 groups of four individuals. 
We tested whether the feeding treatment affected body weight and female fecundity using Welch's ANOVA to account for unequal variances between treatment groups. Sizeassortative mating was tested by comparing the proportion of pairs observed copulating that were homogeneous (i.e. copulations between two well-fed or two poorly fed individuals) with the proportion expected under random mating. This expected proportion was one third given that each individual was offered one potential partner of the same feeding treatment and two potential partners of a different feeding treatment. We computed Pearson's Chi-squared tests based on the proportion of observed and expected homogeneous copulations summed across all social groups.

The used experimental setup might have biased our results given that social groups comprised only four individuals. Specifically, the first copulation in a group might have predetermined all subsequent copulations because as soon as two individuals copulate, the two non-mating individuals have de facto no choice with whom to mate. However, encounters in P. acuta are relatively short compared with the duration of the mating trials of $60 \mathrm{~min}$ (i.e. on average encounters last 9 min in pairs; Janicke et al. 2014) and rare enough (i.e., on average, four copulations per group of four individuals per hour; this dataset) to allow for independent pair formation. Moreover, post-experimental tests revealed that only 16 out of 60 (i.e. $26.7 \%$ ) mating pairs were formed by the two individuals that were not involved in the preceding copulation within the same group, which does not deviate significantly from the expected proportion of $16.7 \%$ under random mating (Pearson's Chi-squared test; $\chi^{2}=1.228, P=0.268, d f=1$ ). Nevertheless, we also tested for size-assortative mating including only data of the first copulation within each pair, which provides a test that is not affected by the potential bias discussed above.

All statistical analyses were carried out in JMP 9.0.3. (SAS Institute Inc. 2010) or in R version 3.1.2 (R Development Core Team 2014). Values are given as means \pm 1 SE unless otherwise stated.

\section{Meta-analysis on size-assortative mating in simultaneous hermaphrodites}

We conducted a meta-analysis on size-assortative mating in hermaphrodites in order to test: (i) whether hermaphrodites usually copulate assortatively with respect to size and (ii) whether our current knowledge is in support of the hypothesis that sexual selection-mediated size-assortative mating is stronger in reciprocally mating species compared to unilaterally mating species. For this, we conducted a literature search using the ISI Web of Knowledge (Web of Science Core Collection, all years) with the 'topic' search terms 'body size and hermaphrod* or size and mating and hermaphrod* or size and pairing and hermaphrod* or size and correlat* and hermaphrod* or size and behav* and hermaphrod*' on 13 January 2015. This resulted in a total number of 935 studies, which we screened for field or laboratory tests of sizeassortative mating in simultaneously hermaphroditic animals. Most of these studies have been excluded on the basis of the information provided in the title $(N=657)$ or in the abstract $(N=201)$. From the remaining 77 studies, we excluded 59 for the following three reasons. First, we excluded studies that did not test for size-assortative mating $(N=52)$. Second, we excluded studies testing for size-assortative mating but for which the presented data clearly indicate that the observed patterns are confounded with spatial differences in body size leading to size-assortative mating that does not result from sexual selection $(N=1$; i.e. Pal et al. 2006 reporting two estimates for the limpet Siphonaria capensis; note that we also excluded one estimate for Physa gyrina reported in DeWitt 1996 for the same reason but this study was included as it also reported reliable estimates for $P$. acuta). Third, we excluded studies comparing mating frequencies between pairs of similar- and differently sized individuals (e.g. Lüscher and Wedekind 2002; Anthes et al. 2006a; Norton et al. 2008), because here the tested individuals are not provided any choice of mating with individuals of different size classes $(N=6)$. This resulted in 18 eligible studies. These studies were checked for cited publications that were not covered by our literature search protocol, which provided one additional estimate of sizeassortative mating (i.e. Crozier 1918).

In total, we obtained 32 effect sizes from 20 studies that tested for size-assortative mating in 18 species (including the two estimates obtained from the empirical tests reported here; see below). Whenever possible, we extracted intra-class correlation coefficients from each study, because this has been argued to be the preferable statistical parameter for evaluating the strength of size-assortative mating (Vreys and Michiels 1997). When intra-class correlation coefficients were not presented, we either converted the provided statistics into $r_{\mathrm{i}}$ (procedure outlined in Lessells and Boag 1987) or extracted the raw data from the graphs provided in the articles using the imaging software GraphClick version 3.0.2 (http://www. arizona-software.ch) and calculated $r_{\mathrm{i}}$ manually. In six cases, we could not extract the required information to compute $r_{\mathrm{i}}$, and therefore, we used the reported Pearson correlation coefficient $r$ instead. Four of these $r$ are based on the correlation of body size between sperm donors and sperm recipients (Tomiyama 1996; Oliver 1997; Chaine and Angeloni 2005); whereas, in two cases, $r$ represents the mean correlation coefficient obtained from randomization tests (Baur 1992). As such, the effect sizes from these studies may overestimate the degree of size-assortative mating (Vreys and Michiels 1997). All correlation coefficients were converted to Fisher's $z$, which served as our effect size used in the final analyses (Borenstein et al. 2009). We classified mating types (i.e. unilateral or reciprocal) according to the 
information provided in the articles (Table S1). Note that the chalk bass Serranus tortugarum and the belted sandfish Serranus subligarius, were classified as a reciprocally mating species even though individuals do not copulate simultaneously in both sex functions sensu stricto. This is because both species exhibit so-called egg trading with both partners exchanging several parcels of gametes sequentially (Petersen and Fischer 1996; Oliver 1997), which has been described as a form of reciprocal mating (Schärer et al. 2014). For P. acuta, we extracted raw data from two studies focussing on sizedependent sex-role preferences (DeWitt 1996; OhbayashiHodoki et al. 2004), which allowed us to compute four effect sizes. In addition, we also included the effect sizes of our study on size-assortative mating of freshly field-caught snails under low and high density.

In order to account for phylogenetic non-independence, we conducted a phylogenetically independent meta-analysis (Lajeunesse et al. 2013). For this, we reconstructed a phylogeny of all sampled species following the methods reported in more detail elsewhere (Chamberlain et al. 2012). In brief, we constructed the topology using published phylogenies (molluscs: Wade et al. 2001; Vonnemann et al. 2005; Dayrat et al. 2011; Gobbeler and Klussmann-Kolb 2011; remaining taxa Dunn et al. 2008). In addition, we added branch length for higher-order taxa using the 'Expert Results' of the public database 'TimeTree' (Hedges et al. 2006) or data published elsewhere (i.e. Lumbricidae: Novo et al. 2011, Serranidae: Betancur-R et al. 2013). We aged undated nodes using the Branch Length Adjuster algorithm (Webb et al. 2008), which sets the age of undated nodes as the midpoint between the two chronologically adjacent nodes to produce an ultrametric tree.

The phylogenetically independent meta-analysis was performed using multivariate linear mixed effects models in the metafor R-package version 1.9.2 (Viechtbauer 2010). This method accounts for nonindependence due to phylogeny including repeated measurements of the same species (i.e. repeated measurements of the same species are assigned a correlation coefficient of 1). First, we ran a restricted maximum likelihood model without any moderator variable to test for size-assortative mating across all species and for heterogeneity among species. We then added mating type (unilateral or reciprocal) as a moderator variable to test our hypothesis that sexual selection-mediated size-assortative mating is stronger in reciprocally mating hermaphrodites. We computed the proportional reduction of the variance components as a pseudo- $R^{2}$ in order to infer the amount of variance explained by mating type (Viechtbauer 2010). Visual inspection of the funnel plot and statistical test for funnel plot asymmetry provided no evidence for a publication bias (Rank-correlation test, $N=32$, Kendall's $\tau=0.112, P=0.372$ ).

\section{Results}

Size-assortative mating in the hermaphroditic freshwater snail $P$. acuta

\section{Size-assortative mating in the field}

Snails differed in shell length between the sectors (ANOVA: $F_{3,132}=12.027, P<0.001$; mean \pm SE for sectors $A$ to $D: A=$ $7.72 \pm 0.18 \mathrm{~mm}, B=8.42 \pm 0.16 \mathrm{~mm}, C=9.29 \pm 0.18 \mathrm{~mm}, D=$ $7.70 \pm 0.21 \mathrm{~mm})$. When neglecting this spatial variation in body size, we observed that size-assortative mating operates in the studied population $\left(r_{\mathrm{i}}=0.297, F_{67,68}=1.844, P=0.006\right.$; Fig. 1a). However, when accounting for spatial differences in shell length by using the standardised estimates, we found no significant evidence for size-assortative mating $\left(r_{\mathrm{i}}=0.092\right.$, $F_{67,68}=1.202, P=0.226$; Fig. 1b).

\section{Size-assortative mating under standardised conditions}

In total, we observed 61 copulations (34 in low-density tanks; 27 in high-density tanks). Pair formation was random with respect to size under both densities (low density: $r_{\mathrm{i}}=-0.199$, $F_{33,34}=0.668, P=0.876$; high density: $r_{\mathrm{i}}=0.240, F_{26,27}=$ 1.632, $P=0.106$; Fig. 2).

\section{Size-assortative mating of experimentally manipulated snails}

The feeding treatment affected body weight and female fecundity (Welch's ANOVA; body weight: $F_{1,46.6}=194.281$, $P<0.001$; number of eggs: $\left.F_{1,44.2}=44.689, P<0.001\right)$. Wellfed snails had a more than 2-fold higher body weight and 3fold higher female fecundity than the poorly fed snails (Figs. 3a, b). Despite these substantial differences between well-fed and poorly fed snails, we found no evidence for size-assortative mating. Summed across all groups, we observed 78 copulations of which 23 (i.e. $29.5 \%$ ) occurred between homogeneous pairs (Fig. 3c), which does not differ statistically from the expected proportion under random mating of one third $\left(\chi^{2}=0.268, P=0.605, d f=1\right)$. Similarly, when restricting the analysis to the first copulation within each group, we found no evidence for size-assortative mating (i.e. only 4 out of 18 copulations were assortative with respect to size; $\chi^{2}=0.554, P=0.457, d f=1$ ). Finally, we assigned all observed copulations to the different mating types including copulations between two poorly fed individuals (PP), between a poorly fed donor and a well-fed recipient (PW), between a well-fed donor and a poorly fed recipient (WP) and between two well-fed individuals (WW). In total, we observed $11 \mathrm{PP}$, $34 \mathrm{PW}, 21 \mathrm{WP}$ and $12 \mathrm{WW}$ copulations, which do not deviate significantly from the expected number of copulations under random mating of $13,26,26$ and 13 , respectively $\left(\chi^{2}=1.805\right.$, $P=0.614, d f=3)$. 
Fig. 1 Correlations of the body size of sperm donors and sperm recipients from copulating pairs sampled in the field. Symbols indicate which of the four different sectors that pairs were sampled from. The shaded areas indicate the $95 \%$ confidence ellipses. Correlations are shown for both raw data (a) and standardised data (b); the latter allowing us to account for spatial heterogeneity in body size (for details, see 'Methods')
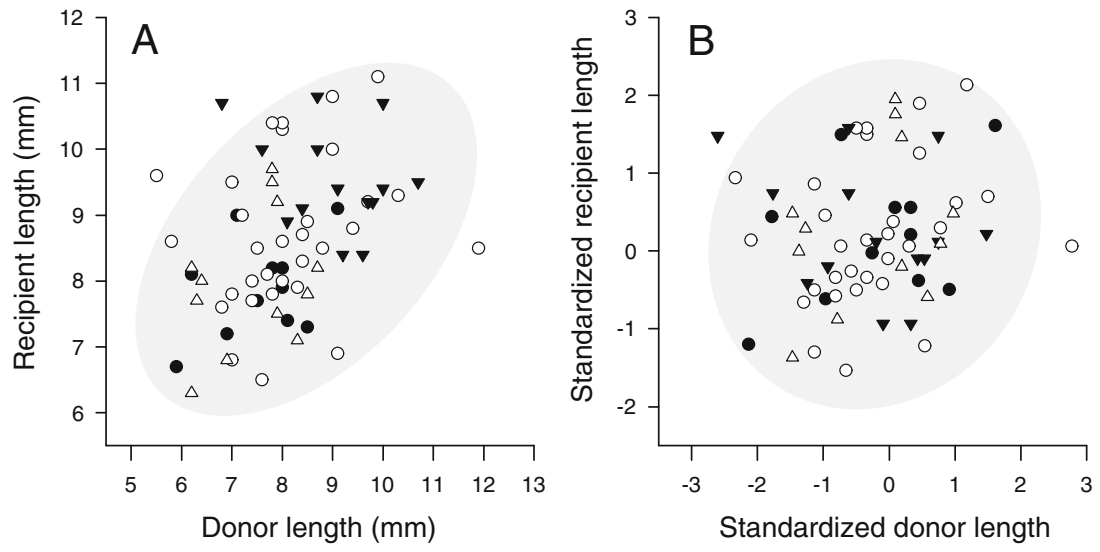

\section{Meta-analysis on size-assortative mating in simultaneous hermaphrodites}

Our meta-analysis suggested that, in general, hermaphrodites copulate assortatively with respect to body size $(z \pm \mathrm{SE}, 0.443 \pm 0.210 ; z$-test: $k=32, z=2.109, P=0.035$; Fig. 4), but we detected substantial and highly significant inter-specific variation in the strength of sizeassortative mating $(Q=144.557, P<0.001, d f=31$; Fig. 4; Table S1). This variation could partly be explained by mating type $\left(Q_{\mathrm{M}}=4.724, P=0.030, d f=1\right.$; pseudo- $R^{2}=0.32$ ). Specifically, size-assortative mating was relatively strong and statistically significant in reciprocally copulating hermaphrodites $(z \pm \mathrm{SE}, 0.508 \pm$ 0.175 ; $z$-test: $k=17, z=2.897, P=0.004)$ but weak and statistically non-significant in unilaterally copulating species $(z \pm \mathrm{SE}, 0.140 \pm 0.219 ; z$-test: $k=15, z=0.641$, $P=0.521)$.

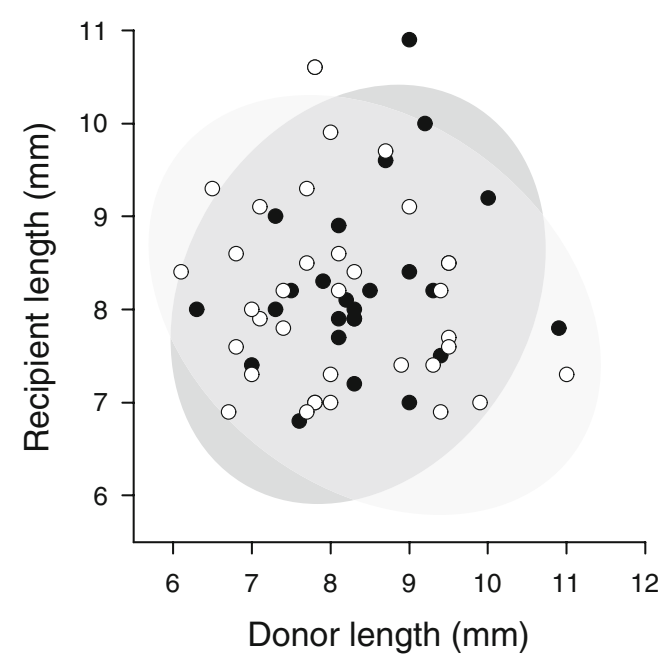

Fig. 2 Correlations of body size between sperm donors and recipients that were observed copulating under high (filled circles) and low (open circles) density conditions. Dark- and light-shaded areas denote the $95 \%$ confidence ellipses for the high- and low-density treatments, respectively

\section{Discussion}

This study provides novel insights on the mating behaviour of hermaphrodites. First, based on three different empirical tests, we show that size-assortative mating based on mating preferences is absent in the unilaterally copulating freshwater snail $P$. acuta. And second, we demonstrate that there is considerable variation in the strength of size-assortative mating among hermaphrodites and that this inter-specific variation can partly be explained by mating type. In the following, we discuss these findings in turn, with a particular focus on illuminating potential methodological pitfalls when testing for sexual selection-mediated size-assortative mating.

\section{Size-assortative mating in $P$. acuta}

Each of the three tests presented in this study provide evidence for an absence of size-assortative mating as a consequence of sexual selection in P. acuta. First, although our raw field data revealed significant size-assortative mating, we demonstrated that this pattern can largely (and potentially completely) be attributed to spatial heterogeneity of body size in the habitat. Body size varied substantially between the arbitrarily defined sectors and it remains unclear to what extent this was due to spatial clustering of snail cohorts differing in age (and thus body size), to size-dependent habitat preferences, to spatial variation in environmental conditions affecting growth or to sampling biases (reviewed in Crespi 1989). Importantly, the significantly positive correlation of body size between two mating partners became much weaker when we statistically controlled for this observed spatial heterogeneity of body size. The remaining tendency (non-significant) toward sizeassortative mating may well reflect spatial heterogeneity in body size at a smaller spatial scale than the one we defined by our four sectors. These findings illustrate that it is very difficult to obtain reliable estimates of the strength of sizeassortative mating mediated by mate choice in the field without detailed knowledge on the spatial heterogeneity of body 


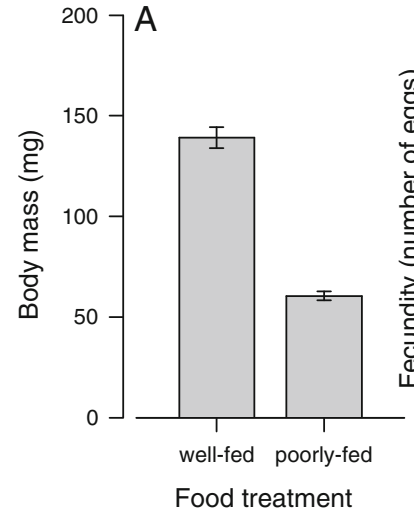

Fig. 3 Test of size-assortative mating using size-manipulated snails. Effects of food manipulation on body weight (a) and female fecundity (i.e. number of eggs; b) and the proportions of homogeneous and heterogeneous pairs observed copulating in groups consisting of two well-fed and two poorly fed snails (c) are shown. In (c), the number of

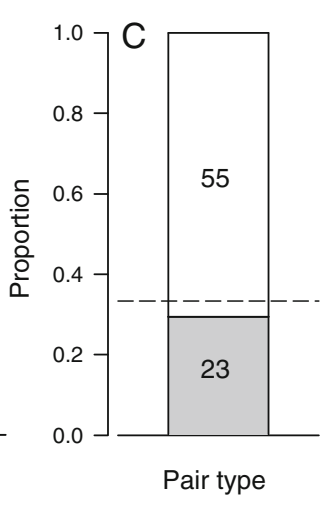

Food treatment

observed homogeneous pair copulations (grey region) and the number of observed heterogeneous pair copulations (open region) are shown. The dashed line indicates the expected proportion of homogeneous pair copulations under random mating with respect to body size (see 'Methods' for details)

Fig. 4 Forest plot and phylogeny used to run the phylogenetically independent meta-analysis of size-assortative mating in simultaneous hermaphrodites. The forest plot shows effect sizes for reciprocally (grey circles) and unilaterally (open circles) mating species. Global effect sizes for each mating type and for all studies combined are shown as filled squares
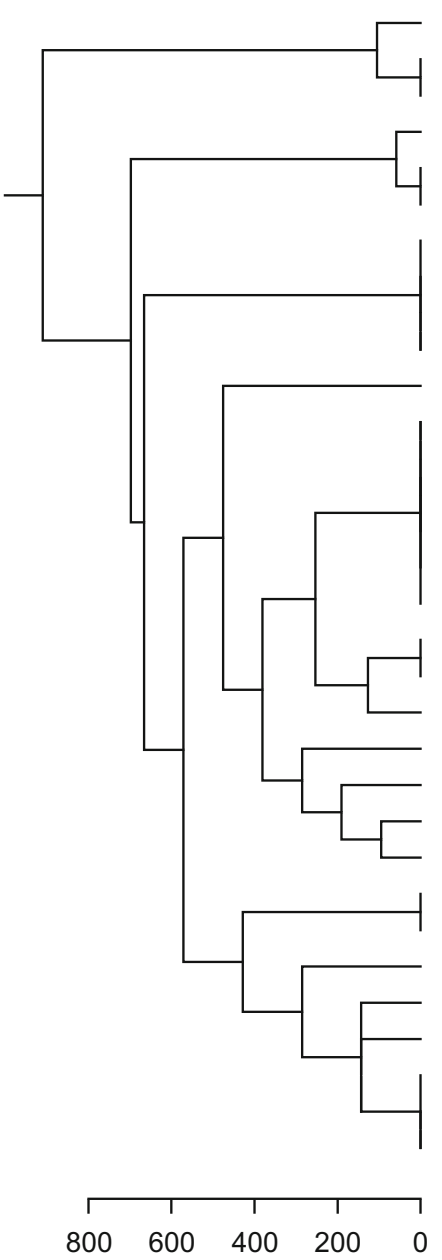

Divergence time

(mya)

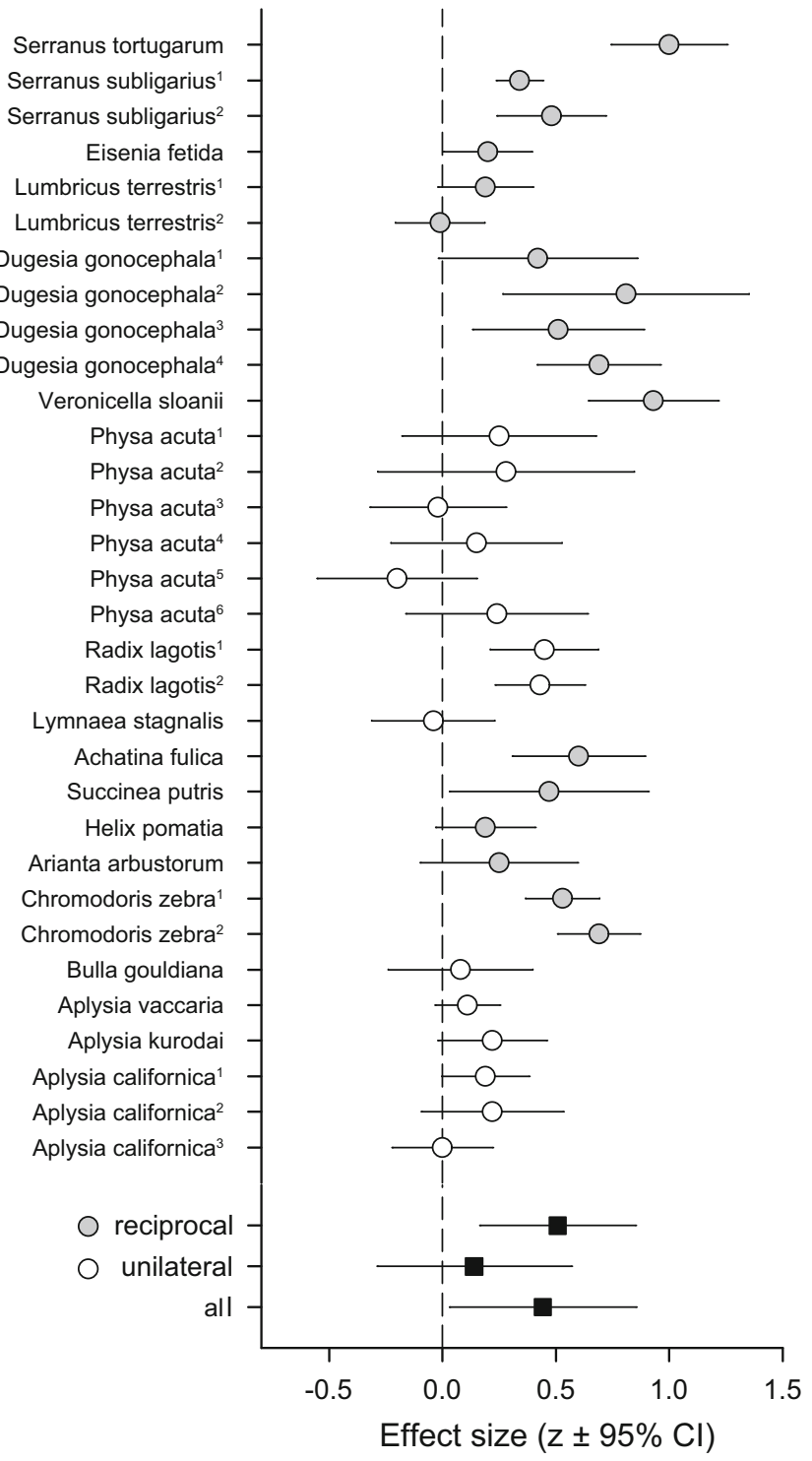


size. Unfortunately, the vast majority of field studies do not consider spatial heterogeneity in body size as a potential confounding factor (e.g. Crozier 1918; Monroy et al. 2005; Clarke and Fields 2013; Yu and Wang 2013; but see Michiels et al. 2001; Pal et al. 2006), and therefore, it is often unclear if observed size-assortative mating is caused by sexual selection or spatial heterogeneity. Consequently, we suggest that future tests for size-assortative mating document spatial differences in body size at the smallest scale that is practical and account for spatial heterogeneity statistically.

An absence of sexual selection-mediated size-assortative mating in $P$. acuta is also supported by our observations of groups of ten freshly field-caught individuals. Size-assortative mating was not detected in either the low-density treatment or the high-density treatment, although there was a slight tendency in the high-density treatment. However, based on the findings of the two other empirical tests presented here (i.e. field study and experimental test of size-manipulated snails) and given the relatively low sample size, we believe that the observed tendency for size-assortative mating under high densities is most likely a type-I error. We are only aware of two descriptive field studies testing for density-dependent size-assortative mating; one in the soldier beetle Chauliognathus pennsylvanicus (McLain 1982) and the other in the milkweed longhorn beetle Tetraopes tetraophthalamus (McLain and Boromisa 1987). Both studies explored size-assortative mating in populations of different densities suggesting a positive effect of density in T. tetraophthalamus and a negative effect in $C$. pennsylvanicus. Given these mixed results, more studies focussing on the effect of density are clearly needed in order to evaluate its potential for explaining intra- and inter-specific variation in size-assortative mating.

The test for sexual selection-mediated size-assortative mating using size-manipulated individuals is presumably the most rigorous of those presented here. This is because specific manipulation allows us to exclude confounding factors such as age (e.g. Nakadera et al. 2015). However, given that we did not manipulate body size directly we still cannot rule out confounding factors that are associated with the feeding treatment. Our food manipulation succeeded in manipulating the body size and the female fecundity of the tested individuals but, despite these differences, pair formation was clearly random with respect to size in groups consisting of two big and two small individuals. Theoretically, our experimental setup might have reduced the strength of size-assortative mating due to the relatively small group size of only four individuals. This is because individuals could have had a preference for mating with a novel mating partner after copulating with an individual of the preferred size, which would lead to an underestimation of size-assortative mating. However, only 24 out of 53 individuals mated more than once and those individuals that copulated repeatedly (i.e. 2 copulations: $N=23 ; 3$ copulations: $N=$ 1) were more likely to copulate again with the same rather than with a novel partner (i.e. only 6 out of 24 individuals copulated with a novel partner). Therefore, we believe that our experimental test of size-assortative mating is not confounded by a preference for novel mating partners under the tested experimental conditions (for mixed results of this socalled Coolidge effect in other freshwater snails, see, e.g. Koene and Ter Maat 2007; Haderer et al. 2009). In sum, the results of our three empirical tests demonstrate unambiguously that size-assortative mating mediated by sexual selection is absent in P. acuta. This result is supported by the two previous studies on size-dependent sex-role preferences in $P$. acuta (DeWitt 1996; Ohbayashi-Hodoki et al. 2004), from which we could extract data and test for size-assortative mating post hoc (see meta-analysis; Fig. 4).

Apparently, P. acuta provides an illustrative example of how, in unilaterally copulating hermaphrodites, a preference for larger mating partners expressed by only one sex function is not enough to promote size-assortative mating. Previous studies have documented that larger individuals have a higher female fecundity (e.g. Janicke et al. 2013; Janicke et al. 2015) and suggested that donors have a preference to inseminate larger partners (Ohbayashi-Hodoki et al. 2004). However, there is currently no evidence for a preference for, or a mating advantage of, larger donors. In fact, male mating success has been found to be size-independent (Pélissié et al. 2012; Janicke et al. 2015) suggesting that a donor's body size is not subject to pre-copulatory sexual selection in terms of mate choice and/or male-male competition.

\section{Size-assortative mating in simultaneous hermaphrodites}

Our meta-analysis revealed three important aspects of sizeassortative mating in simultaneously hermaphroditic animals. First, we found significant size-assortative mating when all effect sizes were combined, indicating that hermaphrodites typically copulate assortatively with respect to size, thus supporting earlier hypotheses (Michiels 1998; Anthes 2010). A recent meta-analysis on size-assortative mating, which was predominantly loaded with estimates obtained from separatesexed organisms, reports a global effect size of $z$ (95\% confidence interval $(\mathrm{CI}))=0.31(0.28-0.35)($ Jiang et al. 2013). This estimate is smaller than our global effect size of $z$ $(95 \% \mathrm{CI})=0.44(0.03-0.85)$, suggesting only a weak tendency for size-assortative mating to be stronger in hermaphrodites compared with separate-sexed species. Unfortunately, Jiang et al. (2013) did not account for phylogenetic nonindependence despite showing that higher taxa differ in the strength of size-assortative mating, which has been demonstrated to lead to erroneous and overconfident estimates of the global effect size (Chamberlain et al. 2012). Hence, the comparison of our estimates of size-assortative mating with the one for separate sexed organisms provided by Jiang et al. (2013) must be treated with caution. 
Importantly, we were able to show for the first time that the variation in the strength of size-assortative mating among simultaneous hermaphrodite species can be partially explained by mating type. As predicted by our hypothesis, sizeassortative mating is stronger in species showing reciprocal mating compared to those with unilateral mating $(z=0.51$ and $z=0.14$, respectively). Species with unilateral matings only showed a weak tendency for size-assortative mating, which was far from statistical significance. Therefore, the apparently stronger size-assortative mating observed in hermaphrodites compared to separate-sexed organisms is solely driven by species that mate reciprocally. In fact, size-assortative mating appears to be weaker in unilaterally mating hermaphrodites than in separatesexed organisms (i.e. $z=0.14$ versus $z=0.31$, respectively), which might be due to conflicts over the preferred sex role (which cannot occur in separate-sexed organisms) that potentially disrupt size-assortative mating in unilaterally mating species.

Even though it seems plausible that mating type affects the strength of size-assortative mating in accordance to our hypothesis, we cannot exclude alternative hypotheses for the observed patterns. First, the causal link between mating type and size-assortative mating can be reversed. Specifically, sizeassortative mating may favour the evolution of reciprocal mating in simultaneous hermaphrodites. This alternative hypothesis assumes that unilateral mating is the ancestral state and that reciprocal mating is advantageous in species with assortative mating (e.g. because it allows more time-efficient copulations in both sex roles). If true, we predict that both sex functions benefit from mating in reciprocally but not necessarily in unilaterally mating species. Second, physical constraints may make size-assortative mating more likely in reciprocal compared to unilateral mating species. This is because reciprocal mating often involves complex postures (e.g. Crozier 1918; Vreys et al. 1997; Schärer et al. 2004), which may require a minimum level of size matching between mating partners to be successful. In this scenario, sizeassortative mating does not result from a preference for mating with larger partners. In fact, this could cause size-assortative mating in reciprocally mating species where neither sex function benefits from mating with larger partners. These alternative explanations highlight the need for more comparative and experimental studies illuminating the interplay between mating type and size-assortative mating in simultaneous hermaphrodites.

Finally, as a third insight from our meta-analysis, there is enormous variation in the strength of size-assortative mating among hermaphrodite species (Fig. 4). Some of this variation presumably reflects methodological differences of how sizeassortative mating was assessed. As discussed earlier, estimates of size-assortative mating obtained from the field are highly sensitive to whether the sampling technique allows spatial or temporal heterogeneity in body size to be statistically accounted for (but note that for our meta-analysis the pooled effect sizes did not differ significantly between field and laboratory studies: $r_{\text {field }}=0.45, r_{\text {laboratory }}=0.39, Q_{\mathrm{M}}=$ $0.273, d f=1, P=0.602)$. Apart from these potential methodological effects, we consider it plausible that some of the observed variation reflects inter-specific differences in the reproductive biology beyond mating type and/or differences in environmental conditions (such as density). Notably, species in which size-assortative mating does not arise from sexual selection might also have contributed to the observed inter-specific variation (reviewed in Crespi 1989; Arnqvist et al. 1996).

We stress the fact that our meta-analysis is based on a relatively small number of studies, which questions the robustness of our conclusions. Therefore, we hope that our study motivates further research on size-assortative mating across many different taxa in order to provide a more powerful test of our hypothesis in the future and to complement our general view of size-assortative mating in simultaneous hermaphrodites. Moreover, further experimental studies (e.g. experimental tests of how environmental conditions affect the strength of sizeassortative mating) and comparative approaches are certainly needed for a better understanding of the factors influencing size-assortative mating in animals.

\section{Conclusions}

This study demonstrates that pair formation in $P$. acuta is random with respect to size when the spatial clustering of similar-sized individuals is controlled for. Therefore we conclude that size-assortative mating as a consequence of mate choice is absent in this unilaterally mating species. Furthermore, our meta-analysis detected significant size-assortative mating across 18 species of hermaphrodites but also revealed substantial interspecific variation in the strength of size-assortative mating. This variation could partly be explained by mating type, providing the first empirical support for the hypothesis that size-assortative mating is stronger in reciprocally compared to unilaterally copulating hermaphrodites. We highlight the need for more empirical and comparative studies that try to explain the interspecific variation in size-assortative mating.

Acknowledgements We are very grateful to Violette Sarda and Nicolas Juillet for providing technical support. We also thank Philippe Jarne and two anonymous reviewers for constructive comments on an earlier version of the manuscript. This project was funded by grants of the Swiss National Science Foundation to T.J. (SNSF grant no: PA00P3-145375/1) and the French National Research Agency to P.D. (ANR ESHAP, ANR12-BSV7-0015). 


\section{References}

Anthes N (2010) Mate choice and reproductive conflict in simultaneous hermaphrodites. In: Kappeler P (ed) Animal behaviour: evolution and mechanisms. Springer, Berlin, pp 329-357

Anthes N, Putz A, Michiels NK (2006a) Hermaphrodite sex role preferences: the role of partner body size, mating history and female fitness in the sea slug Chelidonura sandrana. Behav Ecol Sociobiol 60:359-367

Anthes N, Putz A, Michiels NK (2006b) Sex role preferences, gender conflict and sperm trading in simultaneous hermaphrodites: a new framework. Anim Behav 72:1-12

Arnqvist G, Rowe L, Krupa JJ, Sih A (1996) Assortative mating by size: a meta-analysis of mating patterns in water striders. Evol Ecol 10: 265-284

Baldauf SA, Kullmann H, Schroth SH, Thunken T, Bakker TCM (2009) You can't always get what you want: size assortative mating by mutual mate choice as a resolution of sexual conflict. BMC Evol Biol 9:9

Baur B (1992) Random mating by size in the simultaneously hermaphroditic land snail Arianta arbustorum: experiments and an explanation. Anim Behav 43:511-518

Betancur-R, R., R. E. Broughton, E. O. Wiley, K. Carpenter, J. A. Lopez, C. Li, N. I. Holcroft, D. Arcila, M. Sanciangco, J. C. Cureton Ii, F. Zhang, T. Buser, M. A. Campbell, J. A. Ballesteros, A. Roa-Varon, S. Willis, W. C. Borden, T. Rowley, P. C. Reneau, D. J. Hough, G. Lu, T. Grande, G. Arratia, and G. Orti. 2013. The tree of life and a new classification of bony fishes. PLOS Currents 5

Borenstein M, Hedges LV, Higgins JPT, Rothstein HR (2009) Introduction to meta-analysis. John Wiley \& Sons, Chichester, West Sussex, UK

Chaine A, Angeloni L (2005) Size-dependent mating and gender choice in a simultaneous hermaphrodite, Bulla gouldiana. Behav Ecol Sociobiol 59:58-68

Chamberlain SA, Hovick SM, Dibble CJ, Rasmussen NL, Van Allen BG, Maitner BS, Ahern JR, Bell-Dereske LP, Roy CL, Meza-Lopez M, Carrillo J, Siemann E, Lajeunesse MJ, Whitney KD (2012) Does phylogeny matter? Assessing the impact of phylogenetic information in ecological meta-analysis. Ecol Lett 15:627-636

Clarke N, Fields A (2013) Mating in Veronicella sloanii (Cuvier, 1817) (Veronicellidae). Am Malacol Bull 31:235-244

Crespi BJ (1989) Causes of size-assortative mating in arthropodes. Anim Behav 38:980-1000

Crozier WJ (1918) Assortive mating in a nudibranch, Chromodoris zebra Heilprin. J Exp Zool 27:247-292

David P, Pujol B, Viard F, Castella V, Goudet J (2007) Reliable selfing rate estimates from imperfect population genetic data. Mol Ecol 16: 2474-2487

Dayrat B, Conrad M, Balayan S, White TR, Albrecht C, Golding R, Gomes SR, Harasewych MG, Martins AMD (2011) Phylogenetic relationships and evolution of pulmonate gastropods (Mollusca): new insights from increased taxon sampling. Mol Phylogenet Evol 59:425-437

Development Core Team R (2014) R: a language and environment for statistical computing. R Foundation for Statistical Computing, Vienna

DeWitt TJ (1996) Gender contests in a simultaneous hermaphrodite snail: a size-advantage model for behaviour. Anim Behav 51:345-351

Dunn CW, Hejnol A, Matus DQ, Pang K, Browne WE, Smith SA, Seaver E, Rouse GW, Obst M, Edgecombe GD, Sorensen MV, Haddock SHD, Schmidt-Rhaesa A, Okusu A, Kristensen RM, Wheeler WC, Martindale MQ, Giribet G (2008) Broad phylogenomic sampling improves resolution of the animal tree of life. Nature 452:745-U745
Ferrer M, Penteriani V (2003) A process of pair formation leading to assortative mating: passive age-assortative mating by habitat heterogeneity. Anim Behav 66:137-143

Fischer EA (1984) Egg trading in the chalk bass, Serranus tortugarum, a simultaneous hermaphrodite. Z Tierpsychol 66:143-151

Gobbeler K, Klussmann-Kolb A (2011) Molecular phylogeny of the Euthyneura (Mollusca, Gastropoda) with special focus on Opisthobranchia as a framework for reconstruction of evolution of diet. Thalassas 27:121-154

Haderer IK, Werminghausen J, Michiels NK, Timmermeyer N, Anthes N (2009) No effect of mate novelty on sexual motivation in the freshwater snail Biomphalaria glabrata. Front Zool 6:23

Han CS, Jablonski PG, Kim B, Park FC (2010) Size-assortative mating and sexual size dimorphism are predictable from simple mechanics of mate-grasping behavior. BMC Evol Biol 10:16

Harari AR, Handler AM, Landolt PJ (1999) Size-assortative mating, male choice and female choice in the curculionid beetle Diaprepes abbreviatus. Anim Behav 58:1191-1200

Hedges SB, Dudley J, Kumar S (2006) TimeTree: a public knowledgebase of divergence times among organisms. Bioinformatics 22: 2971-2972

Henry PY, Jarne P (2007) Marking hard-shelled gastropods: tag loss, impact on life-history traits, and perspectives in biology. Invertebr Biol 126:138-153

Hermann PM, Genereux B, Wildering WC (2009) Evidence for agedependent mating strategies in the simultaneous hermaphrodite snail, Lymnaea stagnalis (L.). J Exp Biol 212:3164-3173

Hoefler CD (2007) Male mate choice and size-assortative pairing in a jumping spider, Phidippus clarus. Anim Behav 73:943-954

Honek A (1993) Intraspecific variation in body size and fecundity in insects: a general relationship. Oikos 66:483-492

Janicke T, Schärer L (2009) Sex allocation predicts mating rate in a simultaneous hermaphrodite. Proc R Soc B-Biol Sci 276: $4247-4253$

Janicke T, Vellnow N, Sarda V, David P (2013) Sex-specific inbreeding depression depends on the strength of male-male competition. Evolution 67:2861-2875

Janicke T, Vellnow N, Lamy T, Chapuis E, David P (2014) Inbreeding depression of mating behavior and its reproductive consequences in a freshwater snail. Behav Ecol 25:288-299

Janicke T, David P, Chapuis E (2015) Environment-dependent sexual selection: Bateman's parameters under varying levels of food availability. Am Nat 185:756-768

Jiang YX, Bolnick DI, Kirkpatrick M (2013) Assortative mating in animals. Am Nat 181:E125-E138

Kirkpatrick M (2000) Reinforcement and divergence under assortative mating. Proc R Soc B-Biol Sci 267:1649-1655

Koene JM, Ter Maat A (2007) Coolidge effect in pond snails: male motivation in a simultaneous hermaphrodite. BMC Evol Biol 7:212

Koene JM, Montagne-Wajer K, Ter Maat A (2007) Aspects of body size and mate choice in the simultaneously hermaphroditic pond snail Lymnaea stagnalis. Anim Biol 57:247-259

Kokko H, Rankin DJ (2006) Lonely hearts or sex in the city? Densitydependent effects in mating systems. Philos Trans R Soc B-Biol Sci 361:319-334

Kondrashov AS, Shpak M (1998) On the origin of species by means of assortative mating. Proc R Soc B-Biol Sci 265:2273-2278

Lajeunesse MJ, Rosenberg MS, Jennions MD (2013) Phylogenetic nonindependence and meta-analysis. In: Koricheva J, Gurevitch J, Mengersen K (eds) Handbook of meta-analysis in ecology and evolution. Princeton University Press, Princeton, pp 284-299

Lessells CM, Boag PT (1987) Unrepeatable repeatabilities: a common mistake. Auk 104:116-121

Lüscher A, Wedekind C (2002) Size-dependent discrimination of mating partners in the simultaneous hermaphroditic cestode Schistocephalus solidus. Behav Ecol 13:254-259 
Lynch M, Walsh B (1998) Genetics and analysis of quantitative traits. Sinauer, Sunderland, MA

McLain DK (1982) Density dependent sexual selection and positive phenotypic assortative mating in natural populations of the soldier beetle, Chauliognathus pennsylvanicus. Evolution 36:1227-1235

McLain DK, Boromisa RD (1987) Male choice, fighting ability, assortative mating and the intensity of sexual selection in the milkweed longhorn beetle, Tetraopes tetraophthalmus (Coleoptera, Cerambycidae). Behav Ecol Sociobiol 20:239-246

Michiels NK (1998) Mating conflicts and sperm competition in simultaneous hermaphrodites. In: Birkhead T, Møller AP (eds) Sperm competition and sexual selection. Academic Press, London, pp 219-254

Michiels NK, Hohner A, Vorndran IC (2001) Precopulatory mate assessment in relation to body size in the earthworm Lumbricus terrestris: avoidance of dangerous liaisons? Behav Ecol 12:612-618

Mobley KB, Abou Chakra M, Jones AG (2014) No evidence for sizeassortative mating in the wild despite mutual mate choice in sexrole-reversed pipefishes. Ecol Evol 4:67-78

Monroy F, Aira M, Velando A, Dominguez J (2005) Size-assortative mating in the earthworm Eisenia fetida (Oligochaeta, Lumbricidae). J Ethol 23:69-70

Nakadera Y, Swart EM, Maas JPA, Montagne-Wajer K, Ter Maat A, Koene JM (2015) Effects of age, size, and mating history on sex role decision of a simultaneous hermaphrodite. Behav Ecol 26:232-241

Norton CG, Johnson AF, Mueller RL (2008) Relative size influences gender role in the freshwater hermaphroditic snail, Helisoma trivolvis. Behav Ecol 19:1122-1127

Novo M, Almodovar A, Fernandez R, Giribet G, Cosin DJD (2011) Understanding the biogeography of a group of earthworms in the Mediterranean basin - the phylogenetic puzzle of Hormogastridae (Clitellata: Oligochaeta). Mol Phylogenet Evol 61:125-135

Ohbayashi-Hodoki K, Ishihama F, Shimada M (2004) Body sizedependent gender role in a simultaneous hermaphrodite freshwater snail, Physa acuta. Behav Ecol 15:976-981

Oliver AS (1997) Size and density dependent mating tactics in the simultaneously hermaphroditic seabass Serranus subligarius (Cope, 1870). Behaviour 134:563-594

Pal P, Erlandsson J, Skold M (2006) Size-assortative mating and nonreciprocal copulation in a hermaphroditic intertidal limpet: test of the mate availability hypothesis. Mar Biol 148:1273-1282

Pélissié B, Jarne P, David P (2012) Sexual selection without sexual dimorphism: Bateman gradients in a simultaneous hermaphrodite. Evolution 66:66-81
Petersen CW, Fischer EA (1996) Intraspecific variation in sex allocation in a simultaneous hermaphrodite: the effect of individual size. Evolution 50:636-645

SAS Institute Inc. (2010) JMP v. 9.03., Cary, NC, USA

Schärer L (2009) Tests of sex allocation theory in simultaneously hermaphroditic animals. Evolution 63:1377-1405

Schärer L, Joss G, Sandner P (2004) Mating behaviour of the marine turbellarian Macrostomum sp.: these worms suck. Mar Biol 145: 373-380

Schärer L, Janicke T, Ramm SA (2014) Sexual conflict in hermaphrodites. Cold Spring Harbor Perspectives. doi:10.1101/cshperspect. a017673

Seehausen O, vanAlphen JJM, Witte F (1997) Cichlid fish diversity threatened by eutrophication that curbs sexual selection. Science 277:1808-1811

Tomiyama K (1996) Mate-choice criteria in a protandrous simultaneously hermaphroditic land snail Achatina fulica (Ferussac) (Stylommatophora: Achatinidae). J Mollus Stud 62:101-111

Viechtbauer W (2010) Conducting meta-analyses in R with the metafor Package. J Stat Softw 36:1-48

Vonnemann V, Schrodl M, Klussmann-Kolb A, Wagele H (2005) Reconstruction of the phylogeny of the Opisthobranchia (Mollusca : Gastropoda) by means of $18 \mathrm{~S}$ and $28 \mathrm{~S}$ rRNA gene sequences. J Mollus Stud 71:113-125

Vreys C, Michiels NK (1997) Flatworms flatten to size up each other. Proc R Soc B-Biol Sci 264:1559-1564

Vreys C, Schockaert ER, Michiels NK (1997) Unusual pre-copulatory behaviour in the hermaphroditic planarian flatworm, Dugesia gonocephala (Tricladida, Paludicola). Ethology 103:208-221

Wade CM, Mordan PB, Clarke B (2001) A phylogeny of the land snails (Gastropoda: Pulmonata). Proc R Soc B-Biol Sci 268: 413-422

Webb CO, Ackerly DD, Kembel SW (2008) Phylocom: software for the analysis of phylogenetic community structure and trait evolution. Bioinformatics 24:2098-2100

Wethington AR, Dillon RT (1996) Gender choice and gender conflict in a non-reciprocally mating simultaneous hermaphrodite, the freshwater snail. Physa Anim Behav 51:1107-1118

Yu TL, Wang LM (2013) Mate choice and mechanical constraint on sizeassortative paring success in a simultaneous hermaphroditic Pond Snail Radix lagotis (Gastropoda: Pulmonata) on the Tibetan Plateau. Ethology 119:738-744 